\title{
$t^{4}$ Workshop Report*
}

\section{Integrated Testing Strategies (ITS) for Safety Assessment}

Costanza Rovida ${ }^{1}$, Nathalie Alépée ${ }^{2}$, Anne M. Api ${ }^{3}$, David A. Basketter ${ }^{4}$, Frédéric Y. Bois ${ }^{5}$, Francesca Caloni $^{6}$, Emanuela Corsini ${ }^{7}$, Mardas Daneshian ${ }^{1}$, Chantra Eskes ${ }^{8}$, Janine Ezendam ${ }^{9}$, Horst Fuchs ${ }^{10}$, Patrick Hayden ${ }^{11}$, Christa Hegele-Hartung ${ }^{12}$, Sebastian Hoffmann ${ }^{13}$, Bruno Hubesch ${ }^{14}$, Miriam N. Jacobs ${ }^{15}$, Joanna Jaworska ${ }^{16}$, André Kleensang ${ }^{20}$, Nicole Kleinstreuer ${ }^{17}$, Jon Lalko ${ }^{3}$, Robert Landsiedel ${ }^{18}$, Frédéric Lebreux ${ }^{19}$, Thomas Luechtefeld ${ }^{20}$, Monica Locatelli ${ }^{21}$, Annette Mehling ${ }^{18}$, Andreas Natsch ${ }^{22}$, Jonathan W. Pitchford ${ }^{23}$, Donald Prater ${ }^{24}$, Pilar Prieto ${ }^{25}$, Andreas Schepky ${ }^{26}$, Gerrit Schü̈̈rmann ${ }^{27,28}$, Lena Smirnova ${ }^{20}$, Colleen Toole ${ }^{29}$, Erwin van Vliet ${ }^{30}$, Dirk Weisensee ${ }^{10}$ and Thomas Hartung ${ }^{1,20}$

${ }^{1}$ CAAT Europe, University of Konstanz, Germany; ${ }^{2}$ L'Oréal R\&I, Aulnay, France; ${ }^{3}$ Research Institute for Fragrance Materials, Inc., Woodcliff Lake, USA; ${ }^{4}$ DABMEB Consultancy Ltd, Sharnbrook, UK; ${ }^{5}$ INERIS, DRC/VIVA/METO, Verneuil en Halatte, France; ${ }^{6}$ Università degli Studi di Milano, Department of Health, Animal Science and Food Safety (VESPA), Milan, Italy; ${ }^{7}$ Università degli Studi di Milano, Department of Pharmacological and Biomolecular Sciences (DISFEB), Milan, Italy; ${ }^{8}$ European Society of Toxicology In Vitro, La croix Saint Ouen, France; ${ }^{9}$ National Institute for Public Health and the Environment (RIVM), Centre for Health Protection, Bilthoven, The Netherlands; ${ }^{10}$ CellSystems GmbH, Troisdorf, Germany; ${ }^{11}$ MatTek Corp., Ashland, MA, USA; ${ }^{12}$ Bayer AG, West Haven, USA; ${ }^{13}$ seh consulting + services, Paderborn, Germany; ${ }^{14}$ Cefic LRI and EPAA, Brussels, Belgium; Hubesch Consult BVBA, Sint-Pieters-Leeuw, Belgium; ${ }^{15}$ Scientific Committee and Emerging Risks Unit, European Food Safety Authority, Parma, Italy; current address Centre for Radiation, Chemical and Environmental Hazards, Public Health England, UK; ${ }^{16}$ Procter \& Gamble, Modelling \& Simulation Biological Systems, Brussels Innovation Center, Strombeek-Bever, Belgium; ${ }^{17}$ ILS/NICEATM, Research Triangle Park, NC, USA; ${ }^{18}$ BASF SE, Ludwigshafen, Germany; ${ }^{19}$ Laboratoire de Synthèse Organique, CNRS UMR 7652, Ecole Polytechnique, Palaiseau, France; ${ }^{20}$ Center for Alternatives to Animal Testing (CAAT), Johns Hopkins Bloomberg School of Public Health, Baltimore, MD, USA; ${ }^{21}$ REACH Mastery, Como, Italy; ${ }^{22}$ Givaudan Schweiz AG, Dübendorf, Switzerland; ${ }^{23}$ Departments of Biology and Mathematics, University of York, UK; ${ }^{24}$ Food and Drug Administration, European Bureau, Brussels, Belgium; ${ }^{25}$ EURL ECVAM, Systems Toxicology Unit, Institute for Health and Consumer Protection, European Commission, Joint Research Centre, Ispra, Italy; ${ }^{26}$ Beiersdorf AG, Hamburg, Germany; ${ }^{27}$ UFZ Department of Ecological Chemistry, Helmholtz Centre for Environmental Research, Leipzig, Germany; ${ }^{28}$ Institute for Organic Chemistry, Technical University Bergakademie Freiberg, Germany; ${ }^{29}$ CeeTox Inc., Kalamazoo, MI, USA;

${ }^{30}$ SeCAM Services \& Consultation on Alternative Methods, Agno, Switzerland

\section{Summary}

Integrated testing strategies (ITS), as opposed to a single definitive test or fixed batteries of tests, are expected to efficiently combine different information sources in a quantifiable fashion to satisfy an information need, in this case for regulatory safety assessments. With increasing awareness of the limitations of each individual tool and the development of highly targeted tests and predictions, the need for combining pieces of evidence increases. The discussions that took place during this workshop, which brought together a group of experts coming from different related areas, illustrate the current state of the art of ITS, as well as promising developments and identifiable challenges. The case of skin sensitization was taken as an example to understand how possible ITS can be constructed, optimized and validated. This will require embracing and developing new concepts such as adverse outcome pathways (AOP), advanced statistical learning algorithms and machine learning, mechanistic validation and "Good ITS Practices".

Keywords: in vitro methods, testing strategy, Tox2 lc, skin sensitization, computational toxicology

Received November 1, 2014; accepted November 17, 2014; Epub November 19, 2014;

http://dx.doi.org/10.14573/altex.1411011

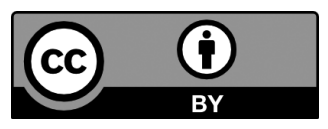

This is an Open Access article distributed under the terms of the Creative Commons Attribution 4.0 International license (http://creativecommons.org/ licenses/by/4.0/), which permits unrestricted use, distribution and reproduction in any medium, provided the original work is appropriately cited.

${ }^{*}$ A report of $t^{4}$ - the transatlantic think tank for toxicology, a collaboration of the toxicologically oriented chairs in Baltimore, Konstanz and Utrecht sponsored by the Doerenkamp-Zbinden Foundation; participants do not represent their institutions and do not necessarily endorse all recommendations made. 


\section{Introduction}

Regulators from different agencies worldwide as well as the regulated scientific community in general are becoming increasingly aware of the limitations of the current safety testing paradigm. Animal-based high-dose testing in typically one stand-alone guideline test is not always relevant for human exposure scenarios. New in vitro and in silico approaches, however, are limited in nature and can usually only either supplement the animal test or serve as one component of a range of information sources that need to be combined in a relevant, reliable and unbiased way (Leist et al., 2014). This is exactly the purpose of the concept of integrated testing strategies (ITS), also known as integrated approaches to testing and assessment (IATA), with the aim to combine different pieces of information / tests in a more mathematically efficient and biologically informed way. For the development of such science-based and human-relevant approaches for safety assessment of chemicals, a decision making process needs to be adopted and accepted for regulatory purposes. In order to better understand the mechanisms and factors involved, it is now well recognized that the future of chemical safety assessment must move away from animal tests towards a combination of complementary approaches (in vitro, ex vivo, in silico, in chemico) that address functional mechanistic endpoints tied to adverse outcomes of regulatory concern. In spite of this increasing shared awareness, the way toward this goal remains unclear. There are controversies surrounding the definition of ITS, extending to how they can be implemented, validated and reach global regulatory acceptance. Results from the different tests need to be combined in an objective and transparent way (Kinsner-Ovaskainen et al., 2009, 2012; Hartung et al., 2013a).

The principle of ITS, as they are used in this context, is to incorporate multiple data from various information streams, derived from different test methods, test method batteries, tiered test schemes, modeling techniques such as (Q)SAR (quantitative structure activity relationship), kinetics, exposure and epidemiological data, HTS (high throughput screening) and computational toxicology, etc. into one decisionmaking process (Judson et al., 2013). In this framework, the role of ITS is crucial, but the way to achieve its aim is not straightforward. There are many challenges: to accommodate the flexibility ITS require, to quantify and respond to varying levels of uncertainty, to incorporate preexisting knowledge, to assess test method availability and reproducibility, to de- fine applicability domains of ITS components or necessary accuracy, all with the requirements of standardization that are mandatory for regulatory applications.

For this reason, there is the need to develop transparent, objective and consistent ITS tools to support reliable hazard and risk assessments. These are the core conceptual ITS requirements formulated in Jaworska and Hoffmann (2010) and later reiterated by Hartung et al. (2013a).

Regarding ITS, there are still many open questions:

- What are the selection criteria for in vitro and in silico methods and the combination criteria of the methodologies for constructing ITS?

- Which statistical and/or mathematical tools are available for relevant integration of data from different sources?

- Can we adopt standards for statistical evaluation?

- How should the predictive performance of ITS be assessed and validated?

- How should the outcome of ITS be evaluated for regulatory use?

To answer these questions, a group of experts was convened, coming from many different areas of expertise and organizations, including regulatory, validation and government bodies (EFSA, EURL ECVAM, US NICEATM, US FDA) and scientific associations (CEFIC, EPAA, ESTIV) (Box 1). The present report represents the outcome of a three-day workshop sponsored and co-organized by CAAT, the Doerenkamp-Zbinden Foundation (DZF), BASF, the International Fragrance Association (IFRA), the Research Institute for Fragrance Materials (RIFM) and the European Society of Toxicology In Vitro (ESTIV). This workshop was held in Ranco (Varese, Italy) on July 8-10, 2013.

\section{Background}

According to the European Chemicals regulation REACH (Regulation EC 1907/2006), safety assessment of a substance is performed through the full characterization of the risks related to its use and distribution, including physical hazards, toxicological and ecotoxicological properties. Those are combined with a detailed exposure assessment for the final definition of the risk management measures that must be implemented for a reasonable safe use, or restriction, of the substance.

The idea of applying multiple testing strategies for hazard and safety assessment started more than twenty years ago (Basketter, 1994). The reasons why a single in vitro test may hardly

Box 1: List of acronyms of organizations with corresponding websites

\begin{tabular}{|c|c|c|}
\hline Acronym & Definition & website \\
\hline CAAT & Center for Alternatives to Animal Testing & caat.jhsph.edu \\
\hline CAAT-Europe & Center for Alternatives to Animal Testing - Europe & cms.uni-konstanz.de/leist/caat-europe \\
\hline CEFIC & European Chemical Industry Council & www.cefic.org \\
\hline DZF & Doerenkamp-Zbinden Foundation & www.doerenkamp.ch/en/?id=10 \\
\hline
\end{tabular}




\begin{tabular}{|c|c|c|}
\hline $\mathrm{ECHA}$ & European Chemical Agency & echa.europa.eu \\
\hline EFSA & European Food Safety Authority & www.efsa.europa.eu \\
\hline EPA & Environmental Protection Agency & www.epa.gov \\
\hline EPAA & European Partnership for Alternative Approaches to Animal Testing & www.epaa.eu.com \\
\hline ESAC & EURL ECVAM Scientific Advisory Committee & see EURL ECVAM \\
\hline ESTIV & European Society of Toxicology In Vitro & www.estiv.org \\
\hline EURL ECVAM & European Union Reference Laboratory for alternatives to animal testing & $\begin{array}{l}\text { ihcp.jrc.ec.europa.eu/our_labs/ } \\
\text { eurl-ecvam }\end{array}$ \\
\hline FDA & Food and Drug Administration & www.fda.gov \\
\hline ICCVAM & $\begin{array}{l}\text { Interagency Coordinating Committee on the Validation } \\
\text { of Alternative Methods }\end{array}$ & ntp.niehs.nih.gov \\
\hline IFRA & International Fragrance Association & www.ifraorg.org \\
\hline JaCVAM & Japanese Center for the Validation of Alternative Methods & www.jacvam.jp/en/ \\
\hline NICEATM & $\begin{array}{l}\text { National Toxicology Program Interagency Center for the Evaluation } \\
\text { of Alternative Toxicological Methods }\end{array}$ & $\begin{array}{l}\text { www.niehs.nih.gov/research/atniehs/dntp/ } \\
\text { assoc/niceatm/ }\end{array}$ \\
\hline $\mathrm{NIH}$ & National Institute of Health & www.nih.gov \\
\hline OECD & Organisation for Economic Co-operation and Development & www.oecd.org \\
\hline RIFM & Research Institute for Fragrance Materials & www.rifm.org \\
\hline RIVM & National Institute for Public Health and the Environment & www.rivm.nl \\
\hline
\end{tabular}

replace a full in vivo test are explained by Natsch (2014), and may be summarized as follows:

1. In vivo processes usually involve a chain of events while one in vitro test often represents only a single or a few steps of this complex process. When the outcome of that chain of events is toxicity, it is referred to as an adverse outcome pathway (AOP).

2. In vitro methods may represent not only a single event (a "key event" in the AOP nomenclature), but also a single mode of action. Different modes of action may cause the same toxicological effect.

3. Different classes of chemicals may require different test methods, e.g., because of the limited applicability domains of some in vitro tests.

4. ADME (absorption, distribution, metabolism and excretion) must be considered; these processes are often not well represented by current regulatory in vitro models.

5. The outcome from an in vitro test may be limited to reflect only a specific in vivo dose response range and more methods are then required to cover the full dose response range.

In spite of the common agreement that ITS hold enormous promise for effective assessment of toxicological properties of chemicals, little has been done to really apply ITS, in the sense of integration of both non-testing (QSAR, read across) and experimental assays. So far, there are very few tools that combine the methods in an objective way, and often the scientific knowledge of many toxicity mechanisms is still not available or at least not clear enough to apply a full testing strategy that provides certainty that the endpoint is fully covered.
In recent years, many initiatives have started and several papers have been published that foster the application of ITS. The first legal implementation of the ITS concept was the approval of the REACH Regulation (Regulation EC 1907/2006). This ground-breaking legislation is the first in which the combined application of non-standard procedures for safety assessment was included in a legal text (see Annex XI). Starting from Article 1 , there is explicit reference to the possibility of applying alternative methods to avoid new tests on animals. Annex XI of the Regulation explains how non-standard procedures can be used, with explicit mention of the Weight of Evidence (WoE) approach, defined as the conclusion derived "from several independent sources of information leading to the assumption/conclusion that a substance has or has not a particular dangerous property, while the information from each single source alone is regarded insufficient to support this notion." The concept was further extended in the series of guidelines that followed the REACH publication (http://echa.europa.eu/support/guidance), which explain how to use a testing strategy for each endpoint, e.g., how to use new tests for the definition of substance properties, and through the EU FP6 project OSIRIS that was devoted to developing ITS schemes for human and environmental endpoints (http://www.ufz.de/osiris). Within the scope of REACH, WoE can be defined as the organization of existing information while the set up of an ITS is the decision process that leads to performing new tests.

A vast number of chemicals have been registered within the REACH program and if each of them were tested in vivo then the costs in terms of animal lives and economic resources 
would be enormous (Hartung and Rovida, 2009; Rovida and Hartung, 2009). Mid 2014, about 12,500 individual substances had been registered within the scope of REACH and most of the data are available in a public database accessible from the ECHA website (http://echa.europa.eu/information-onchemicals/registered-substances). This database represents a vast resource of structured information that may feed models as well as offering the possibility to check the outcome of various predictions. Methods by which registrants tried to apply non-standard approaches are regularly reported by ECHA, the European Chemicals Agency (ECHA, 2014).

EURL ECVAM plays a significant role: Recently, the 3T3 Neutral Red Uptake Cytotoxicity Assay for Acute Oral Toxicity received a positive opinion from the EURL ECVAM Scientific Advisory Committee (ESAC) for the identification of substances with an $\mathrm{LD}_{50}>2000 \mathrm{mg} / \mathrm{kg}$ with the caveat that, due to the limitations of the test method, results should always be used in combination with other information sources to build confidence in the negative assay results. EURL ECVAM fully endorsed the ESAC opinion and further recommended the development of ITS aiming at full or at least partial identification of acute oral toxicity hazard according to the GHS (Categories 1 to 4 ) (http://ihcp.jrc.ec.europa.eu/our_labs/eurl-ecvam/eurl-ecvamrecommendations/3t3-nru-recommendation).

In the past, the area of skin and eye irritation yielded interesting results with proposed strategies that may fully replace in vivo testing (Scott et al., 2010). EURL ECVAM organized a workshop on validation of ITS that gave the opportunity for a very interesting discussion among scientists (KinsnerOvaskainen et al., 2012). It was proposed that there is no need for formal validation of ITS for screening purposes, for risk assessment purposes and for hazard classification and labelling unless there is an intention to replace a test in use for regulatory purposes.

In response to both the new Regulation for cosmetics products (Regulation EC 1223/2009), which prohibits new tests on living vertebrate animals for cosmetic purposes, and to answer the demands of consumers, who are more and more responsive to animal welfare aspects, academia, cosmetics industries as well as chemical suppliers have been very active with large investments and research programs. Cosmetics Europe is now cofunding with the European Commission the cluster of projects called SEURAT-1 (http://www.seurat-1.eu), which is investing significant resources to find how repeated dose toxicity studies can be replaced by alternative methods. This large project initiative combines five research projects, a central data and knowledge management project and a multidisciplinary coordination action team, trying to integrate advanced techniques in the area of stem cells, microfluidic bio-reactors, in silico modelling, etc.

The application of ITS for the assessment of skin sensitization potential of chemicals is an area of focus of EPAA (European Partnership for Alternative Approaches to Animal Testing, a voluntary collaboration between the European Commission and representatives from both industry and trade associations), with the aim of improving and implementing the 3 Rs approach in the regulatory framework. EPAA organized two workshops to further elaborate how to apply testing strategies for skin sensitization (Basketter et al., 2013). During the ITS-focused EPAA workshop (September 26, 2011, Basketter et al., 2012a), all participants agreed on the idea that ITS was necessary to improve safety assessment and not "just" a way to save animals. In that workshop it was also proposed to further promote the involvement of regulators in order to expedite acceptance of the new approach. Regulatory involvement may also represent a stimulus for the industry to use new methods as soon as possible. In fact, the subsequent EPAA workshop was hosted by ECHA in Helsinki (February 4, 2013), with almost 60 participants from industry, ECHA, EURL ECVAM, OECD (Box 1) and many European Member State representatives (Basketter et al., 2013).

A quantitative WoE approach has been developed through the OSIRIS project with ITS schemes for skin sensitization, mutagenicity and carcinogenicity employing Bayesian networks (Buist et al., 2013; Rorije et al., 2013), keeping in mind that the latter is one of several opportunities for handling situations of redundant and conflicting information. Beyond aquatic endpoints, which are out of the scope of the present paper, the OSIRIS project produced interesting results in the area of repeated-dose toxicity (Tluczkiewicz et al., 2013) and reviewes opportunities for predicting physico-chemical properties in the regulatory context (Nendza et al., 2013).

The above-mentioned projects and activities provide examples of developments taking place mainly in the EU. In 2007, the National Research Council of the US National Academy of Sciences published the well-known report on Toxicity Testing in the $21^{\text {st }}$ century (NRC, 2007), which explained why the classical approach to toxicology assessment was not adequate to cope with present-day needs. Traditional approaches were found to be too time-consuming and expensive, requiring also the sacrifice of many animals and, worst, most in vivo studies may not reflect the human response. The revolution in this approach to toxicity testing is to investigate the possible mechanisms of action of the chemical substances on human cells and human gene targets to better predict the human response. Following this concept, the EPA's ToxCast research program is testing thousands of chemicals in a broad array of cellular, in vitro, biochemical and in silico models, thereby biologically phenotyping a large number of substances via a huge number of endpoints (Tab. 1). Phase I of ToxCast tested predominantly food-use pesticides that already had a wealth of animal toxicity data from regulatory guideline studies. The results were used to create computational models to predict endpoints such as developmental and reproductive toxicity (Martin et al., 2011; Kleinstreuer et al., 2011; Knudsen, 2012). Phase II and Phase III vastly expanded the chemical libraries to cover many untested compounds, providing the opportunity to validate the predictive signatures and prioritize environmental chemicals for potential hazards (http:// epa.gov/ncet/toxcast/data.html).

The work program of OECD, the umbrella organization for chemical testing harmonization representing 34 countries worldwide, is also relevant in this context. OECD work on test 
Tab. 1: List of work packages in the ToxCast and Tox21 HTS projects Further details at http://www.epa.gov/ncct/toxcast/chemicals.html

\begin{tabular}{|l|l|l|l|l|l|}
\hline Set & Chemicals & Assays & Endpoints & Completion & Available to Tox21 partners \\
\hline ToxCast Phase I & 293 & $\sim 600$ & $\sim 1100$ & 2011 & $03 / 2013$ \\
\hline ToxCast Phase II & 767 & $\sim 600$ & $\sim 1100$ & $03 / 2013$ & $10 / 2013$ \\
\hline ToxCast Phase IIla & 1001 & $\sim 100$ & $\sim 100$ & ongoing & $2015 ?$ \\
\hline E1K (endocrine) & 880 & $\sim 50$ & $\sim 120$ & $03 / 2013$ & $10 / 2013$ \\
\hline Tox21 & 8,193 & $\sim 25$ & $\sim 50$ & ongoing & ongoing \\
\hline
\end{tabular}

guidelines and Good Laboratory Practice (GLP) is crucial and generally well accepted as a standard for regulatory purposes. The OECD series on testing and assessment, No.168 (OECD No. 168, 2012a,b) is particularly interesting as it states the possibility of approaching the assessment of an endpoint, in this case skin sensitization, by applying the concept of an AOP that represents the existing knowledge concerning the linkage between a molecular initiating event and an adverse outcome at the individual or population level (Ankley et al., 2010). The OECD idea is that combinations of mechanistically based test methods within IATA are needed to be able to substitute the regulatory animal tests currently in use. Recently, OECD published a new guidance document describing an IATA for skin corrosion and irritation (OECD No. 203, 2014). This is the first well-defined IATA that is being adopted by the OECD. Notably, OECD is also responsible for the OECD QSAR Tool Box (http://www. qsartoolbox.org), which is freely available software that helps risk assessors to identify structural alerts and define groups and similarities among chemicals. Even though not directly focused on ITS, this software represents an interesting tool that can assist users in building optimized ITS, and the OECD is actively working to further develop such aspects for IATA, in the hazard assessment and test guideline programs.

\section{Comparison of the European and American approaches}

ITS is a very generic term, and there are different ways in which ITS may be constructed, e.g., with many tests/substances casting a wide net vs. a priori network construction and assay development. Their construction may differ also according to the starting point, i.e., beginning from the definition of a relevant endpoint (top down) or for screening a wide array of substances (bottom up). The EU (Basketter et al., 2012b) vs. US approach (Kavlock et al., 2012) reflects such a difference: The driving force in the EU stands on new regulations that explicitly ask for application of in vitro tests before performing any new in vivo studies, with the ultimate example being the new regulation of cosmetic products stipulating the complete ban of tests on living animals for cosmetics products and ingredients. In contrast, the US approach driven by Tox $21 \mathrm{c}$ and the ToxCast project (Tab. 1) evaluates many chemicals on a set of assays that should address the different pathways of toxicity (PoT) and modes of action (MoA) in humans. Testing is supposed to produce data that must be interpreted and then compared to a threshold determination for adversity. This approach is different from the European idea of first studying in detail the mode of action and then developing tests that mimic each step of the process.

A constructive way to compare and combine the two approaches may lie in testing a common set of chemicals, such as the OECD reference chemicals developed for various test guidelines, and the ToxCast library. The ECHA database (http:// echa.europa.eu/web/guest/information-on-chemicals/registered-substances) is particularly useful because it contains data about all registered substances within the scope of the REACH program. Provided the data are well prepared and reliable, this will facilitate a better understanding of the applicability domain of the relevant assays.

\section{Composition of ITS}

The term ITS has been generally used when more than one test is applied in combination to characterize the toxicological effects of a substance. ITS were initially conceived with the idea of replacing in vivo tests, with the awareness that no single in vitro test can reproduce the complex interactions that occur in an intact organism. Some pioneering work was done by the ECVAM Integrated Testing Taskforce, which presented ITS as a combination of toxicodynamic and toxicokinetic parameters (Blaauboer et al., 1999). Even though that principle is still valid, nowadays the aim of ITS is definitely broader, with the ambition that ITS must help elucidate the mechanism of action of chemical perturbations with respect to human health or the environment. Ideally, ITS will be the highest quality source of mechanistic information for the definition of safety assessment.

Such evolution in the concept of ITS explains why there is not a unique definition and why there are many ways to combine tests to build a testing strategy, as explained in Figure 1. The two simplest forms of ITS are a battery of tests and tiered strategies, as explained by Jaworska and Hoffmann (2010). A battery of tests is executed in parallel and generally the results of all tests are necessary for the definition of a specific property. In tiered strategies series of tests are applied in se- 
A: Weight of evidence

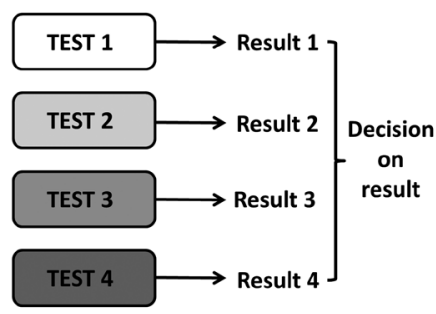

B: Battery of tests

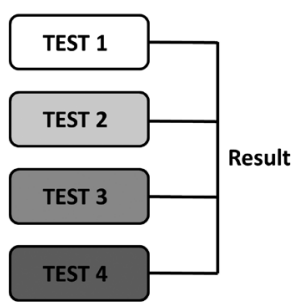

C: Tiered strategy

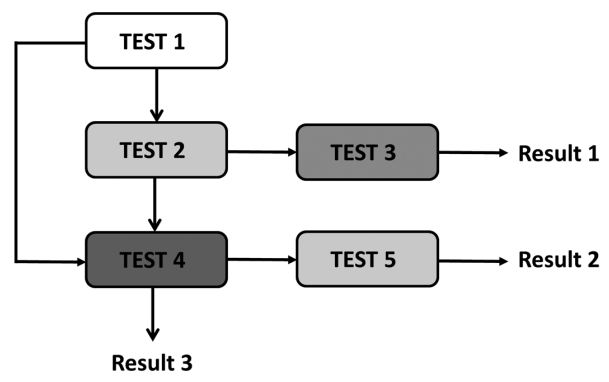

Fig. 1: Schemes for the different approaches that are considered as form of ITS

A, Weight of Evidence (WoE). A set of independent assays provides the same number of results. None of them alone is sufficient to make a decision, but all together may lead to the final decision on the endpoint. This approach is described in Annex XI of REACH and considered as acceptable from a regulatory point of view, even if none of the tests is performed in a standard way.

$\mathrm{B}$, Battery of tests. The final result is defined by the sum of the results from many tests that all must be performed. This approach implies that all methods share the same applicability domain. It is considered by many scientists as the only possibility for in vitro methods to replace in vivo tests.

$\mathrm{C}$, Tiered strategy. According to the results of the first step it is decided which following test must be performed. It is an open system as there is no precise combination of tests, which may be in vitro methods or QSAR evaluations. Any of the tests can return either a number or a mechanistic elucidation or a yes/no answer.

The three schemes are not always well separated. In some cases, not all tests from a battery (Fig. 1B) are necessary for the definition of the endpoint, while in some cases the path of a tiered strategy (Fig. 1C) is considered mandatory, resembling more a battery of tests. Stopping and decision making is triggered when a predefined knowledge level is reached.

quence, by following yes or no decisions, as is the example of eye irritation (Balls et al., 1999), or a chain of events, as in the case for skin sensitization (Maxwell et al., 2011; Van der Veen et al., 2014; Natsch, 2014). The most advanced Bayesian decision methods are based on such a tiered strategy but take into account the fact that test results are not perfect "yes/no" answers and that within this information value there is some uncertainty; the overall uncertainty decreases as the weight of the results accumulates. More generally, the ITS should be composed of building blocks with precise decision points that can halt the process when a pre-defined level of confidence is reached and should optimize the number of tests that are required accordingly. The $\mathrm{WoE}$ approach is usually based on existing data while ITS should prospectively address which assays need to be performed for the assessment of an endpoint or in general for the definition of the risk posed by the use of a substance. However, the ITS concept has some similarity to the WoE approach as it represents a way to combine different results to make a decision (Balls et al., 2006).

ITS are very context-dependent and multiple solutions are likely to be available and desirable. Explicitly, an ITS should contain the following elements:

1. Information target identification;

2. Systematic exploration of knowledge;

3. Choice of relevant inputs;

4. Methodology to synthesize disparate evidence;

5. Methodology to guide testing
Generally speaking, ITS should combine different building blocks, which can also be based on non-test (in silico and readacross) and test methods, and the final decision about the safety assessment of a substance should be based on information from more than one type of source.

All results acquired through the testing must have quantifiable confidence levels and associated uncertainties to enable the application of an objective probabilistic approach. Welldeveloped and widely used probabilistic modeling tools are the Bayesian Networks (BN), whose potential in ITS framework was introduced by Jaworska and Hoffmann (2010), although other techniques are also feasible (Jaworska et al., 2013). Classification algorithms, such as classification and regression trees (CART) and random forests, were recently used to construct testing strategies for acute oral toxicity testing (KoppSchneider et al., 2013; Prieto et al., 2013; Kinsner-Ovaskainen et al., 2013).

Though it is clear that not all steps need to be measured for safety assessment, setting of decision points is not easy and depends upon how much information is required to fulfill the specific needs. This is highly dependent on the purpose of the ITS, ranging from simple hazard identification and chemical prioritization to a sophisticated risk assessment with increasing numbers of tests and levels of complexity. For example, in some cases the measure of chemical reactivity, e.g., with the DPRA (Direct Peptide Reactivity Assay), can be regarded as sufficient as a yes/no answer for a simple preliminary hazard categoriza- 
Tab. 2: Comparison between the possible composition of an ITS strategy that can be immediately applied and the innovative ITS that should be pursued for a more efficient safety assessment

\begin{tabular}{|l|l|}
\hline ITS for hazard characterization $(\mathrm{REACH})$ & ITS for full safety assessment \\
\hline Prescribed & Flexible \\
\hline Deterministic & Probabilistic \\
\hline Classification & Fit for purpose \\
\hline
\end{tabular}

tion of the substance (Gerberick et al., 2007). In the context of the EU FP6 project ACuteTox, the estimation of the oral LD50 dose from an effective concentration in vitro and the application of classification algorithms were used to predict official acute oral toxicity categories (Prieto et al., 2013).

Ultimately, any ITS should be designed to fit a specific purpose, by balancing the applicability domain of the tests, sufficient information, cost and experimental feasibility. Using acute oral toxicity as an example, Norlen and colleagues (2014) compared the cost-effectiveness of different approaches based on single methods (four in silico tools and one in vitro cytotoxicity assay) and battery combinations of methods. They nicely illustrated how to assess the cost-effectiveness of alternative methods and how to interpret the results.

Another difficulty that may arise when a combination of tests is proposed for the prediction of an adverse effect, comes from the fact that the majority of in vitro tests are developed independently, as "stand-alone" prediction models for hazard identification. Most method developers still hope to find the perfect in vitro method to fully replace an in vivo test, in spite of the fact that in reality usually a set of complementary in vitro tests is necessary to reflect complex endpoints.

Each assay that contributes to building the ITS has to be well characterized, whether it is based on the biochemical understanding of the MoA, cellular effects or is related to other aspects that, for example, may impact the bioavailability of the substance. Test method definitions should include a defined protocol, the precise scope of the final endpoint, information on applicability domain and the variability of the measurements. The proper test selection varies according to the context of the testing strategies. In some applications, e.g., for cosmetics, sensitivity or reliable identification of no toxicity is more important than specificity, but this can be different for other uses of the substance, for example if there is relevant exposure.

The feasibility of in vitro testing may be limited; a variety of different tests may be necessary in order to cover the full chemical universe and chemical properties, for example, the applicability domain is limited in case of poorly water-soluble substances.

The definition of the predictive capacity is even more challenging: when many results are combined, each of them brings its own variability with an impact on the definition of the toxicity threshold.

Another fundamental aspect is the kinetics of the effect and the relationship between the concentrations tested in vitro with respect to doses of the organism exposure. It is necessary to consider the absorption, distribution and the metabolism of the substance in the human organism, the metabolism and interaction among organs (Yoon et al., 2012; Jacobs et al., 2013). This fundamental aspect is often underestimated. For example, there are accepted methods for the analysis of skin penetration, but few that consider the actual permeance of a substance in the epidermis, where the sensitization process starts (Basketter et al., 2007). In general, calculation related to the in vivo distribution of the substance and its metabolites should be considered. This aspect is essential to underpin the so-called quantitative in vivo/in vitro extrapolation (QIVIVE) (Blaauboer et al., 2012; Bessems et al., 2014).

The way forward for ITS is outlined in Table 2. An innovative ITS should be flexible and preferably based on a probabilistic approach; assays should be selected to gain the best balance between the number of tests (i.e., effort, time and resources) and information that is obtained. All single inputs, in chemico, in silico and/or in vitro, must be combined in a way that acknowledges and statistically assesses their respective contributions. Economic considerations may have to be considered too, as usually methods that are more complex are also more expensive, requiring sophisticated equipment and technical expertise.

ITS must be adaptive, allowing straightforward omission or addition of new tests as they become available or when newly acquired knowledge yields a more effective combination. At any level, the reasoning for any selection must be transparent, objective and independent of the personal judgment of the operators.

The statistical tools used to interpret ITS outcomes must be objective and able to evaluate when the acquired knowledge has reached a sufficient level of confidence to fit the final purpose and stop the process, moving from a deterministic approach with a preconceived belief in the ability to perfectly foresee the effect to a probabilistic prediction of the final outcome on the human population. Ideally, in the future, informatics tools may provide value of information analyses that identify the next tests to run in order to reach the final goal of optimizing costs, resources and predictive accuracy. Even if the $\mathrm{BN}$, for example, is a promising informatics tool for combining the different components of the ITS, this methodology needs formal approval, and novel network learning tools may be applied to free the process from personal judgment. 


\section{The example of skin sensitization}

While a broad applicability of ITS is expected for many toxicological endpoints, skin sensitization was chosen here as a welldeveloped example and test case of how ITS can be applied in practice. The biological mechanism of skin sensitization is well known, the concepts for replacing animal testing for this hazard have been developed in a series of projects (Rovida et al., 2013; Van Loveren et al., 2008; Maxwell et al., 2011), and there are many in vitro methods that are either validated or at an advanced state of validation, as well as a validated in vivo method (Local lymph node assay, LLNA) that may serve as reference (NIH, 1999).

The chains of events that trigger the sensitization response are now sufficiently understood (Fig. 2). For a chemical to induce skin sensitization a number of events must take place and some of them are considered to be key events, essential for the adverse outcome. Physical and chemical properties of the substance are important as only substances of low molecular weight (LMW) overcome the skin barrier. As LMW substances are too small to cause an immunogenic reaction, chemical allergens must bind to extracellular and cellular skin proteins to form a complete antigen (hapten binding). Protein binding is considered as the molecular initiation event (MIE) of the AOP. Following uptake of the complete antigen and in the context of danger signals primarily secreted by activated keratinocytes, dendritic cells (DC) mature and migrate via the afferent lymph vessels to the regional lymph nodes. In the lymph nodes, mature DC (expressing cell surface markers such as CD80 or CD86) stimulate the activation of hapten-specific responsive $\mathrm{T}$ cells, leading to the generation of Tc1 effector cells. Upon renewed contact with the same chemical, memory $\mathrm{T}$ cells are recruited to the site of contact. Interactions between T cells and antigen presenting cells can take place in the skin, thus initializing the inflammatory reaction (elicitation phase). This sequence of events is started by the hapten, while the substance itself may represent a pre/pro hapten, i.e., it can be either metabolized or modified by any physico-chemical agent on the skin.

This chain of events was chosen by OECD to illustrate the concept of AOP (OECD No 168, 2012a,b), i.e., a description of existing knowledge concerning the linkage(s) between a MIE and an adverse outcome at the individual or population level (Fig. 3). A specific substance can be tested with different methods, each representing one or more steps in the AOP. Some of these assays are still at an early stage of development, while others are much more advanced in the validation process (Fig. 4).

With regard to skin sensitization, existing information (in vitro, in vivo and/or human data) combined with in silico data (readacross, QSARs) can be sufficient to reach a decision for the intended aim, e.g., hazard identification. A possible step forward is a tiered approach beginning with analyzing chemical reactivity with the DPRA, followed by data from cell based assays, such as either the Keratinosens ${ }^{\mathrm{TM}}$ (Natsch et al., 2011) or the human Cell Line Activation Test (hCLAT, Sakaguchi et al., 2009). This may yield a consistent prediction and then be sufficient, or it may result in data conflict, requiring more testing to resolve the conflict and arrive at a final conclusion. Such a simple approach may answer the question of hazard identification, i.e., yes/no sensitizers, and continuing with other tests, by considering the migration of the dendritic cells or $\mathrm{T}$ cell reaction, may become necessary if better definition of the minimum threshold to trigger human

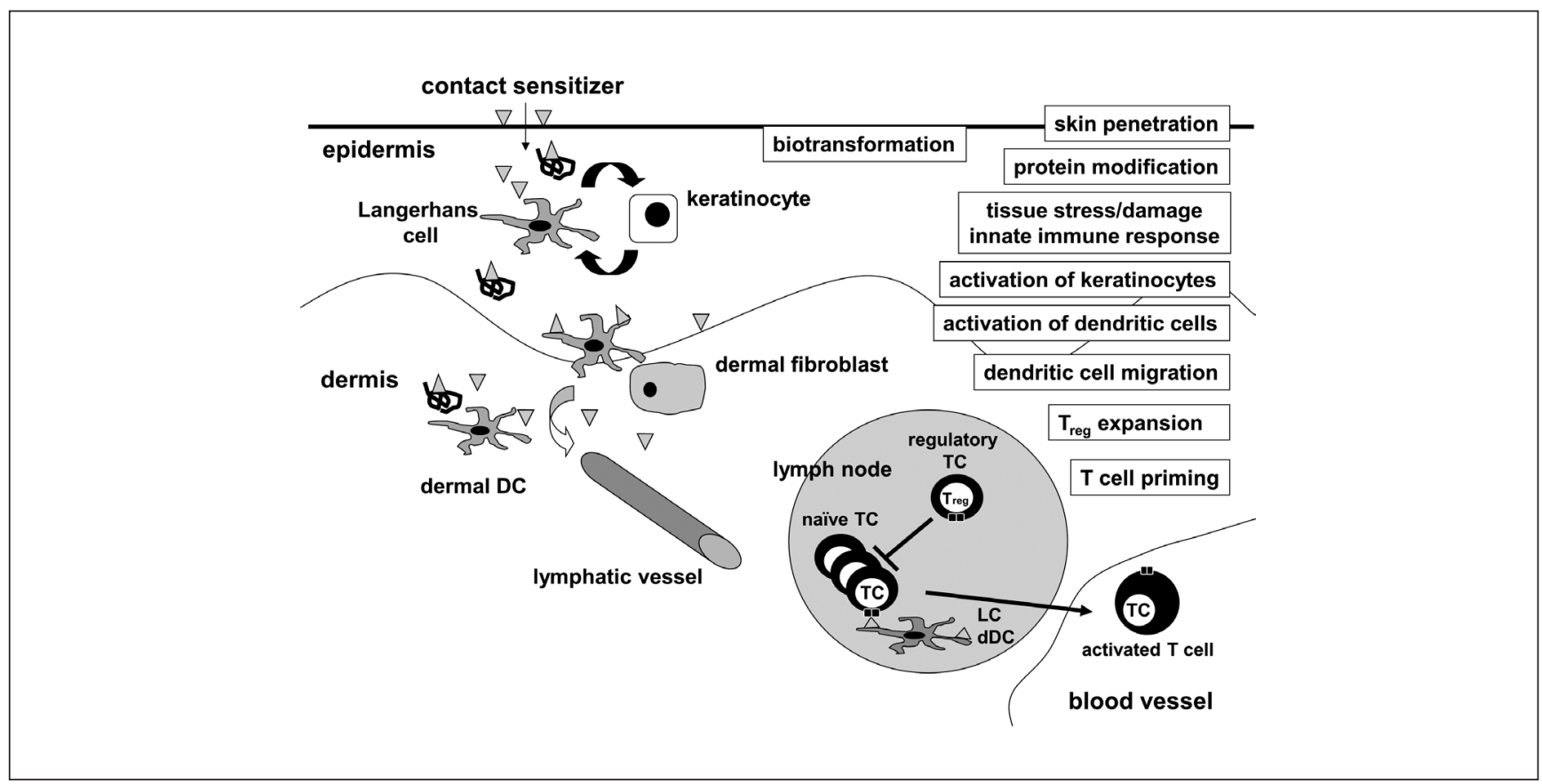

Fig. 2: Scheme of the sequence of events that may induce skin sensitization potential

From Rovida et al. 2013 


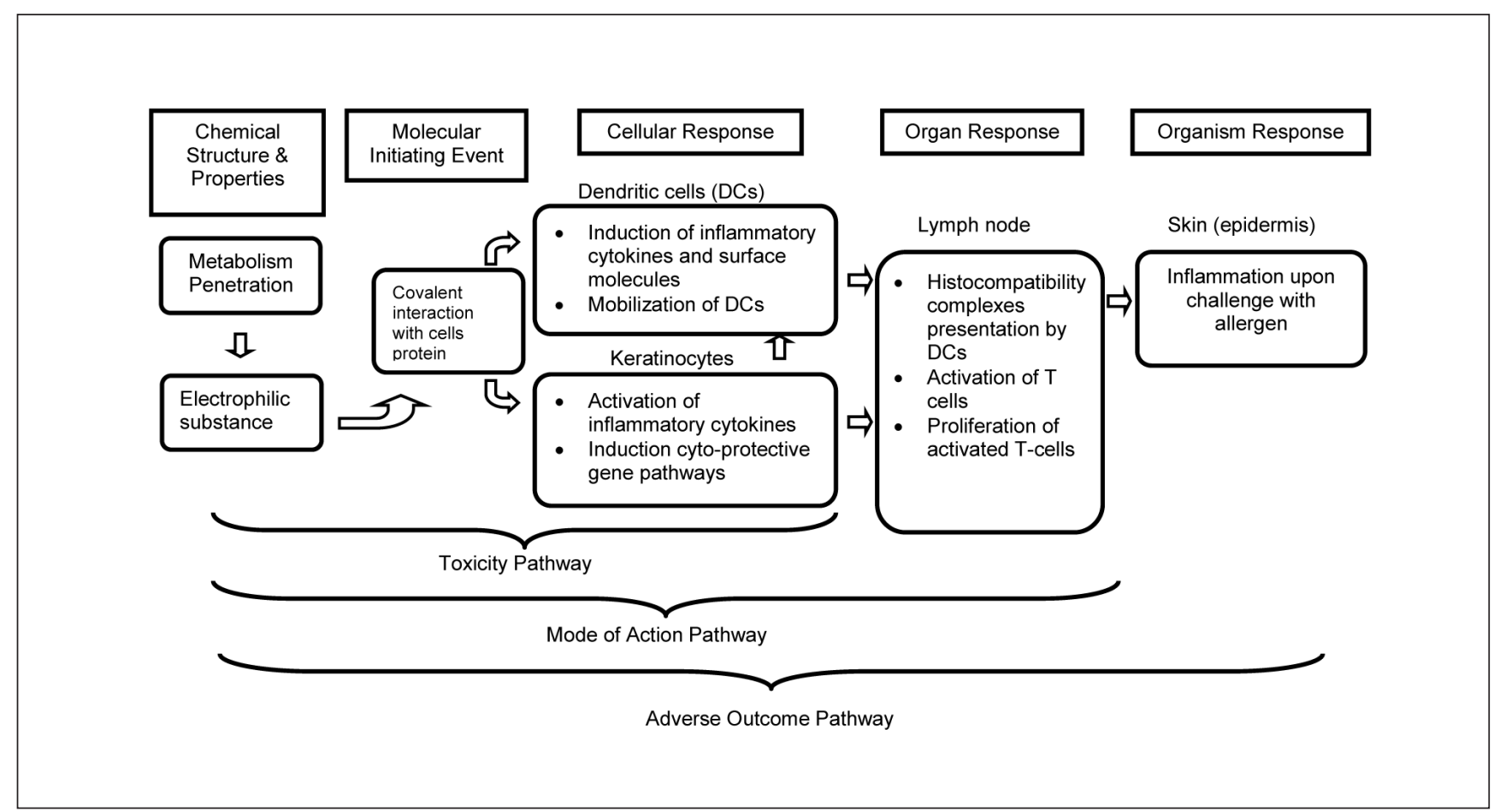

Fig. 3: Flow diagram of the pathways associated with skin sensitization

From OECD No 168a-b, 2012, reproduced with kind permission.

\section{ADVERSE OUTCOME PATHWAY}

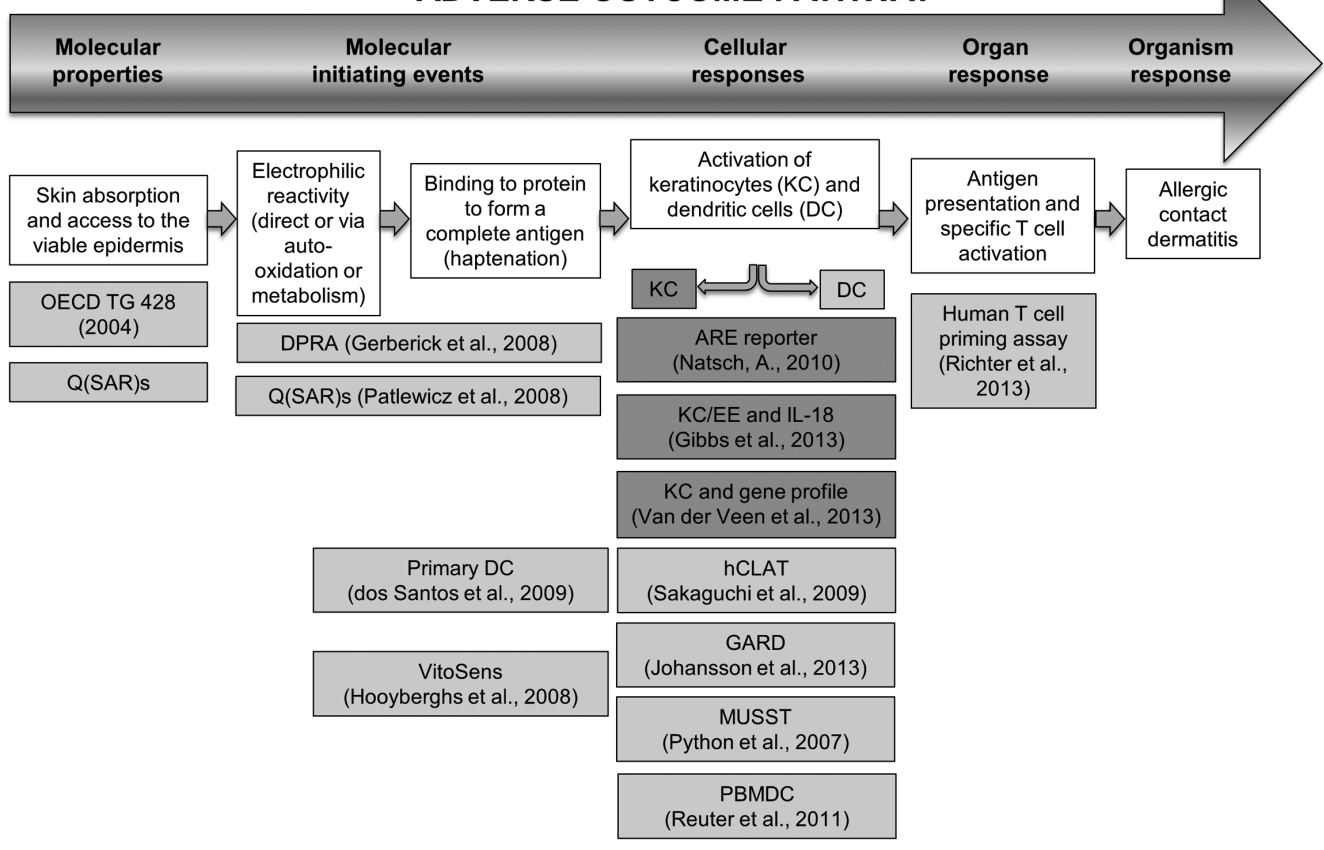

Fig. 4: Adverse outcome pathway with potential non-animal methods for contact hypersensitivity

References provide explanations on methods' names, principles and protocols. The methods listed here are non-exhaustive and are meant as examples only; other methods are available and may also be applicable. 
reaction is required or to distinguish between skin and respiratory sensitizers (Rovida et al., 2013). Mathematical models have been proposed to quantitatively establish a relationship between the dose of sensitizer applied to the skin and the possible human adverse effect (Maxwell et al., 2014).

Recently, EURL ECVAM published its recommendation on the DPRA ${ }^{1}$ and the Keratinosens ${ }^{\mathrm{TM}} 2$ assays; for hCLAT also a DB-ALM (EURL ECVAM DataBase service on ALternative Methods) protocol was published by ECVAM ${ }^{3}$. The performance of each method is measured in isolation, with its own reproducibility, reliability and relevance assessment, to ensure that it will be sufficiently robust for test guideline development. Accuracy has been calculated for the set of chemicals tested as a preliminary evaluation only. Recent OECD documents (OECD No. 168, 2012a,b) explain which methods will enter the strategy, and an ITS for skin sensitization is being finalized. Because the OECD membership is comprised of many different regulatory frameworks, precise instructions as to how to quantitatively integrate the results may need flexibility, depending upon the regulatory jurisdiction.

It has been demonstrated (Bauch et al., 2012; Natsch et al., 2013; Van der Veen et al., 2014) that the proper and appropriate combination of those methods may increase the final predictivity with respect to skin sensitization hazard identification, and that this approach may even exceed the accuracy of the LLNA. Also, potency estimates for skin sensitization from ITS have been presented (Jaworska et al., 2013). This was possible because many chemicals are well characterized with regard to their sensitization properties. In future this can be even further expanded by considering the corresponding human response (Basketter et al., 2014). While highlighting the importance of defining a set of reference substances for ITS development, this fact also highlights the difficulties in compiling a proper list of reference substances for other endpoints, such as reproductive toxicity, (developmental)neurotoxicity (Smirnova et al., 2014) and so on, when only in vivo animal data are available.

\section{Validation of ITS}

Validation of alternative methods usually refers to the modular approach introduced by Hartung et al. (2004). Traditionally, the validation procedure is based on the evaluation of a single method in comparison to the traditional method, which is considered as a reference standard. Validation of in vitro methods is now undertaken by EURL ECVAM in the EU and by ICCVAM (Box 1) in the US, with important contributions from JaCVAM (Box 1) in Japan and other OECD member countries, under the auspices of the OECD.

Establishing precise rules for ITS validation may be complex, as the ITS itself is a dynamic process that cannot be defined by strict rules and may in fact be designed to provide an evaluation of the mechanistic validity of the approach. Nevertheless, the assessment of any ITS must be rigorous, objective and transparent, with scientific validity, which must fit the purpose. This procedure, even though not considered a formal alternative method validation, must be endorsed by an official organization to facilitate regulatory acceptance and recognition by government institutions.

For that purpose, some specific rules need to be defined. First, each test of the strategy must be standardized, with assessment regarding reproducibility and transferability of the protocol with a clear definition of the applicability domain and the uncertainty of the measurement. Moreover, the type of information that each method delivers should be clear, whether it is to demonstrate a mechanistic action, a physico-chemical property, or to elucidate a PoT or AOP. As ITS are inherently evolutionary (i.e., undergoing constant refinement over time), the scientific validity of the approach needs to be ensured, where applicable, with a regular peer-review process.

A problem arises when the final performance of an ITS has no defined parameters and references for comparison, as is the case for complex endpoints such as neurotoxicity or reproductive toxicity. In those cases, a step-by-step procedure may represent the solution. The precise definition of the endpoint and the mechanistic drivers is the first step, followed by a proposed description of the AOP. The knowledge of the in vivo mechanism is essential even though it is not necessary that each and every step of the in vivo process is represented by a test. The opposite is true, i.e., the mechanistic relevance of the specific assay must be defined together with the demonstration that it fits the purpose in the strategy, which should be clearly defined, whether it is for hazard, for potency or for the identification of a single PoT.

Validation is based on the reproducible and accurate response of the strategy when challenged with a set of chemicals with well-known characteristics and therefore the reference chemical selection is essential for a successful process. Those chemicals must have a broad array of structural characteristics in terms of physical and chemical properties, e.g., octanol/water partition coefficient $\left(\mathrm{K}_{\mathrm{ow}}\right)$, mechanism / mode of action (if known) plus toxicological behavior that should include the whole range of activity with both positive and negative controls. The compilation of a set of reference standards can be useful as exemplified for sensitization (Casati et al., 2009; Kolle et al., 2013) as the starting point for the development of new methods or for the comparison of two equivalent methods. Extensive validation will still require a larger number of chemicals. Active consultations with statisticians is important to decide on the proper number of chemicals and datasets needed to have statistically sufficient power, considering prevalence but also the purpose of the strategy (Kopp-Schneider et al., 2013). At this moment this is probably the main constraint, because the list of chemicals with known toxicological profiles really relevant to humans is very limited. Most of the time, only results from animal studies are

\footnotetext{
1 http://ihcp.jrc.ec.europa.eu/our_labs/eurl-ecvam/eurl-ecvam-recommendations/eurl-ecvam-recommendation-on-the-direct-peptide-reactivity-assay-dpra

2 http://ihcp.jrc.ec.europa.eu/our_labs/eurl-ecvam/eurl-ecvam-recommendations/recommendation-keratinosens-skin-sensitisation

3 http://ecvam-dbalm.jrc.ec.europa.eu/view_doc.cfm?iddoc=1558\&tdoc=prot
} 
available, and the relevance in translating these studies to human biology is generally questionable.

The applicability domain of the ITS must also receive special attention, being derived from the overlap of the applicability domains of each single assay composing the strategy. Foreseeing exchangeable building blocks may also help to enlarge the applicability domain of the whole strategy, for example by including different assays for either water or non-water soluble materials.

The definition of the predictive capacity is definitely more challenging for several reasons. In particular, an ITS aims to give an answer as to the final effect rather than simply attempting to reproduce the performance of an animal test. However, the desired predictivity should be defined according to the final use (e.g., cosmetic or industrial chemicals, drugs, pesticides, etc.) and the effect that it is intended to predict, whether it is for hazard identification, classification and labelling, potency or even the capacity to exclude a specific risk.

Another difficulty lies in the mathematical combination of tests that return many different types of results, ranging from binary outcomes to multi-dimensional continuous results, and ultimately to the explanation of a mechanism. The analysis of many chemicals with known behavior is fundamental but not always possible. While there is much data on sensitization, data is sparse for other endpoints such as reproductive toxicity or neurotoxicity. In this sense, the validation of the mechanism is preferable to a validation based on the final results obtained with single chemicals (Hartung et al., 2013b).

Finally, yet importantly, the evaluation of the robustness of the strategy is essential. This can be achieved through the requirement that each test must meet specific minimum intra- and inter-laboratory reproducibility, which need to be known and integrated in the strategy. A combination of the building blocks may influence the overall robustness of the strategy, considering that the uncertainty of the measure may propagate when more methods are included. The final uncertainty is not only a matter of propagation of the error of each test; more careful and comprehensive sensitivity analyses are needed. Each assay may have a unique and variable impact on the final outcome and provide information that may range from dichotomous to continuous. For this reason, a straightforward approach is preferable considering that unambiguous definitions may clear the way for regulatory acceptance.

A good tool to guide the process is provided by the principles of evidence based toxicology (EBT) with calls for transparency, objectivity and consistency of approaches (Hoffmann and Hartung, 2006; Guzelian et al., 2005).

\section{Discussion}

In spite of the awareness of the scientific community of the need for ITS as a tool for safety assessment, its full applicability is still constrained by several factors including lack of agreement on the approach, testing methods that are not developed for the purpose and the lack of a validation procedure for regulatory acceptance. To overcome these difficulties, some precise prerequisites must be defined.
First of all, each single component of an ITS has to be defined. The purpose of each test must be clear, the protocol precise, and its robustness and variability must be carefully quantified. Even if each of those steps has to be adapted to the specific test, the general principle of scientific evidence has to be preserved.

Those pre-requisites can be immediately applied when rigid tiered strategies are used by combining validated test methods, as is done, for example, for eye irritation (EPA, 2009), acute oral toxicity (Prieto et al., 2013) or more recently for developmental toxicity (Sogorb et al., 2014) and reproductive toxicity (Piersma et al., 2013), even though these are still at a proposal stage. Despite the large investment of time and resources toward providing a straightforward and transparent approach, even the case of skin sensitization is complex, with the ambition of combining tests for bioavailability, mechanistic elucidation, reactivity and potency.

Beyond assessing the validity of each single test, the whole procedure must have precise and objective characteristics for regulatory acceptability. It can be imagined that in the future, ITS will probably define the overall safety assessment of a substance, rather than the single effect, such as skin sensitization, acute systemic toxicity, developmental toxicity and so on.

From the perspective of ideal ITS as shown in Figure 5, the final results must be independent from any subjective interpretation. This can be achieved by setting minimum requirements for an ITS tool. However, the necessity for precision, objectivity and reproducibility of the ITS should not impair the necessary characteristic of ITS adaptability. Adaptability of ITS includes the possibility of introducing new tests whenever available and to adapt the ITS to the specific purpose for which it is applied. In fact, the end of the process is defined by the purpose and this can range from mechanistic elucidation to hazard identification

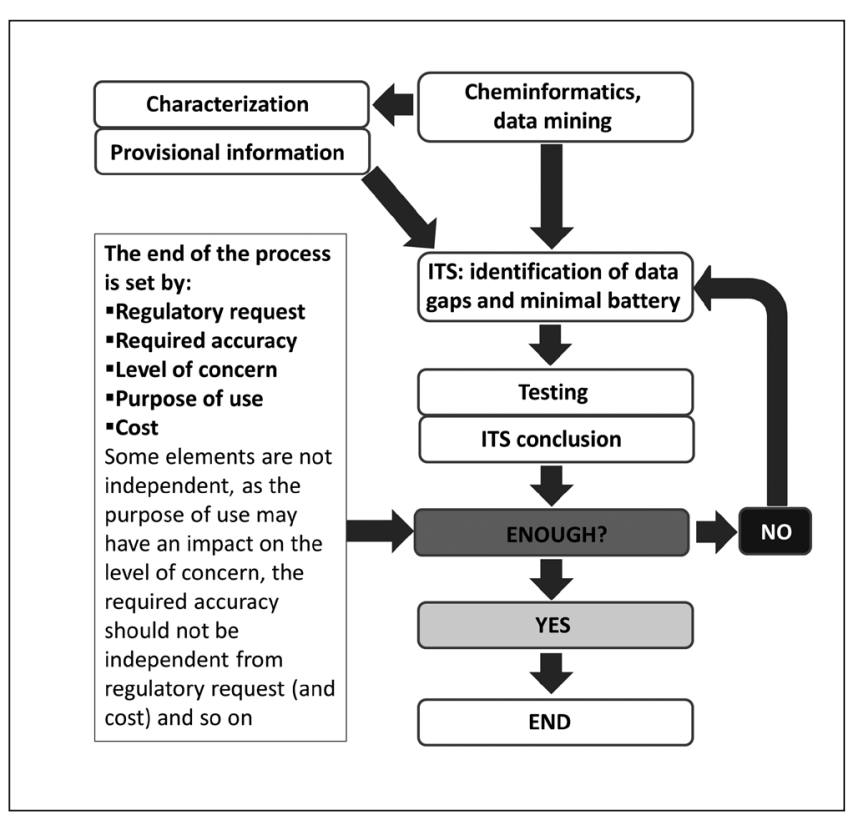

Fig. 5: Scheme for an optimal ITS

Each step should be ruled by precise and objective decisions. 
to a full definition of complex endpoints for specific regulatory purposes.

Learning from the example of QSAR in regulatory use, the principle for validation was defined by OECD through 5 principles ${ }^{4}$ :

1) a defined endpoint

2) an unambiguous algorithm

3) a defined domain of applicability

4) appropriate measures of goodness-of-fit, robustness and predictivity

5) a mechanistic interpretation, if possible

The same principle could be translated to ITS validity, with some adjustments of the concepts. It should be remembered that a QSAR approach may be one of the components of the ITS and as such it must follow the principles defined by OECD.

The list below tries to develop conceptual requirements for ITS, some of them already formulated in Jaworska and Hoffmann (2010):

\section{1) A defined endpoint}

The intent of a defined endpoint in the scope of QSAR should be translated to a clearly defined purpose when referring to ITS, with the awareness that more than one route is possible to get to the same result. Regarding ITS, the concept of a defined purpose is more relevant than the endpoint. This is very important as any ITS may have different levels of complexity in accordance with the intended outcome. The defined purpose is necessary for the decision on when the ITS process can stop.

\section{2) An unambiguous algorithm}

The algorithm that is applied to combine the different components of the ITS must be transparent and reproducible with defined tracking of any changes. The informatics and statistical tools that are applied to get to a final decision must be clearly identifiable through proper and accessible documentation. Compared to QSAR, the algorithm for ITS has another dimension of complexity as it requires adaptability to incorporate results from new tests with minimal delay in adopting new approaches. The respective software programs should include transparent and documented decision rules.

\section{3) A defined domain of applicability}

Each test of the ITS must have a defined applicability domain. The final result should always be within the limitation posed by each component. The concept of an applicability domain should be enlarged to include the purpose of the ITS in addition to the simple decision whether a specific chemical belongs to the applicability domain of the set of tests.

\section{4) Appropriate measures of goodness-of-fit, robustness and predictivity}

This requirement is common to all scientific experimental procedures. In the case of using existing data, possibly derived from non-standard methods, some sort of adjustment to combine the results should be applied. Currently, this is typically based on expert judgment that can evaluate the validity of the data based on the available information. As more and more analyses are performed according to strict quality criteria, this will allow more independent and objective evaluations.

\section{5) A mechanistic interpretation, if possible}

Mechanistic interpretation is the key to accepting non-standard methods that usually lack points of reference. The mechanistic interpretation is commonly linked to the experimental protocol that is supposed to elucidate the specific PoT rather than the whole ITS. ITS based on a fully defined sequence of an AOP are most promising, i.e., they best reflect the key events of the AOP step by step through singular in vitro/in silico methods.

If these five principles are applied, the quality of the final ITS will be unquestionable. However, it should be recognized that we are currently working in an intermediate situation, where there is the need to apply ITS even though in some cases they are still under development.

Regarding ITS execution, a quality system should be immediately implemented in addition to the experimental work. The concepts presented here lay the framework for defining a set of procedures that constitute Good ITS Practice (GIP), similar to what has been done for Good Laboratory Practice (GLP), Good Manufacturing Practice (GMP), Good Cell Culture Practice (GCCP), etc. The concept of GIP was not extensively discussed during the workshop and it will not be further expanded here.

\section{Conclusions and recommendations}

The route toward ITS for hazard and risk assessment is still long and difficult, with undefined conditions for success. In spite of that, some steps should be immediately implemented:

\section{Establishment of an international task force}

It is evident that the scientific community is pursuing different approaches. Probably, the largest difference is between the US, where the approach of Tox $21 \mathrm{c}$ has mainly resulted in large data generation and data-mining aims to identify the most informative tests (ultimately for ITS), and the EU where there is the aim to fully reproduce in vitro the steps that lead to an adverse outcome in vivo. As is always the case, both approaches have advantages and drawbacks. The creation of a supranational task force may improve the dialogue to inform and benefit from each approach, by also exploiting the results obtained on both sides. It is very important to communicate while being open minded to accept improvements, wherever they come from. We propose to create a transatlantic task force that regularly meets to discuss the improvements and helps to implement the lessons learnt overseas.

4 http://www.oecd.org/env/ehs/risk-assessment/37849783.pdf 


\section{Establishment of a general database}

There are already many databases in different fields and many large companies have their own databases, plus the ECHA database, which contains information for all REACH registered substances. Unfortunately, most databases have different formats and the data is not easily extracted; this represents a hindrance, but does not prevent their use within the framework of ITS. A new platform should permit the use of data while respecting confidentiality claims and preserving intellectual property rights, including datasets which are not fully publicly visible. The usefulness of such a database is measured by the success in encouraging scientists to fill it with their data. Scientific funding bodies might provide economic compensation for such additional efforts to the benefit of the scientific community.

\section{Definition of performance standards for ITS}

Special attention should be given to the identification of reference substances that probably represent the main bottleneck to the acceptability of ITS. Validation of alternative methods is now performed using animal tests as the reference standard, but this procedure is not applicable to ITS which strive to demonstrate the true risk for human health. This is very important, in particular in those areas where animal models are weak, such as developmental toxicity, systemic toxicity, etc. The definition of the minimal number of chemicals that must be included in the set of reference substances is an aspect to be considered with the aid of bio-statisticians. The relevant structural diversity of the chemicals selected needs to be carefully considered at both the biological and computational model level.

\section{Mechanistic validation}

At the moment, mechanistic validation is the only proposed scientifically rigorous possibility to overcome the problem of traditional validation vs. non-validated and non-relevant animal models. Validation of ITS should move towards a mechanistic validation to demonstrate that the crucial mechanism causing the damage to human health as a consequence of the action of a xenobiotic is reflected. Mechanistic validation is the answer, even though details about how to apply it are not yet defined, representing another subject for discussion among experts.

\section{Set up Good ITS Practices (GIP)}

For quality assurance, GIP should be the very first step towards setting the rules for ITS creation and implementation, creating a common language that will tremendously improve data-sharing and acceptability. A team of experts should define a list of rules for GIP, following evidence-based principles. It can thereby become a scientific assessment paving regulatory acceptance. Part of GIP should be the establishment of a standardized method to record and track versions of the IT tools that are applied, listing all variations and improvements.

\section{Training and education}

Training and education are always central to the adoption of new scientific approaches (Daneshian et al., 2011). In particular, ITS are an emerging topic where consolidated experience be- longs to relatively few experts, while the majority of risk assessors are still anchored to the traditional approach. Teaching the principles of ITS should be widely disseminated in universities together with the organization of practical training courses. At the moment there are many initiatives but they all rely upon the personal drive of certain individuals. A more harmonized and widespread program should be established to achieve a common understanding.

As a conclusion of the workshop, it is clear that much work is needed to reach the goal of completely replacing animal testing with integrated and mechanistically based testing strategies. There was, however, agreement that the fundamental elements are in place. Toxicologists agree on the general focal principles and efforts needed to develop precise sets of guidelines and practical tools that enable applicability of ITS for safety assessment.

\section{References}

Ankley, G. T., Bennett, R. S., Erickson, R. J. et al. (2010). Adverse outcome pathways: a conceptual framework to support ecotoxicology research and risk assessment. Environ Toxicol Chem 29, 730-741. http://dx.doi.org/10.1002/etc.34

Balls, M., Berg, N., Bruner, L. H. et al. (1999). Eye irritation testing: The way forward; the report and recommendations of ECVAM workshop 34. ATLA 27, 53-77.

Balls, M., Amcoff, P., Bremer, S. et al. (2006). The principles of weight of evidence validation of test methods and testing strategies. The report and recommendations of ECVAM Workshop 58. ATLA 34, 603-620.

Basketter, D. A. (1994). Strategic hierarchical approaches in acute toxicity testing. Toxicol In Vitro 8, 855-859. http:// dx.doi.org/10.1016/0887-2333(94)90086-8

Basketter, D. A., Pease, C., Kasting, G. et al. (2007). Skin sensitisation and epidermal disposition: The relevance of epidermal disposition for sensitisation hazard identification and risk assessment. The report and recommendations of ECVAM workshop 59. ATLA 35, 137-154.

Basketter, D. A., Crozier, J., Hubesch, B. et al. (2012a). Optimised testing strategies for skin sensitization - the LLNA and beyond. Regul Toxicol Pharmacol 64, 9-16. http://dx.doi. org/10.1016/j.yrtph.2012.06.003

Basketter, D. A., Clewell, H., Kimber, I. et al. (2012b). A roadmap for the development of alternative (non-animal) methods for systemic toxicity testing. ALTEX 29, 3-89. http://dx.doi. org/10.14573/altex.2012.1.003

Basketter, D. A., Alépée, N., Casati, S. et al. (2013). Skin sensitization - moving forward with non-animal testing strategies for regulatory purposes in the EU. Regul Toxicol Pharmacol 67, 531-535. http://dx.doi.org/10.1016/j.yrtph.2013.10.002

Basketter, D. A., Alépée, N., Ashikaga, T. et al. (2014). Categorization of chemicals according to their relative human skin sensitising potency. Dermatitis 25, 11-21. http://dx.doi. org/10.1097/DER.0000000000000003

Bauch, C., Kolle, S. N., Ramirez, T. et al. (2012). Putting the 
parts together: Combining in vitro methods to test for skin sensitising potentials. Regul Toxicol Pharmacol 63, 489-504. http://dx.doi.org/10.1016/j.yrtph.2012.05.013

Bessems, J. G., Loizou, G., Krishnan, K. et al. (2014). PBTK modelling platforms and parameter estimation tools to enable animal-free risk assessment: recommendations from a joint EPAA-EURL ECVAM ADME workshop. Regul Toxicol Pharmacol 68, 119-139. http://dx.doi.org/10.1016/j. yrtph.2013.11.008

Blaauboer, B. J., Barratt, M. D. and Houston, J. B. (1999). The integrated use of alternative methods intoxicological risk evaluation ECVAM integrated testing strategies task force report 1. ATLA 27, 229-237.

Blaauboer, B. J., Boekelheide, K., Clewell, H. J. et al. (2012). The use of biomarkers of toxicity for integrating in vitro hazard estimates into risk assessment for humans. ALTEX 29, 411-425. http://dx.doi.org/10.14573/altex.2012.4.411

Buist, H., Aldenberg, T., Batke, M. et al. (2013). The OSIRIS Weight of Evidence approach: ITS mutagenicity and ITS carcinogenicity. Regul Toxicol Pharm 67, 170-181. http://dx.doi. org/10.1016/j.yrtph.2013.01.002

Casati, S., Aeby, P., Kimber, I. et al. (2009). Selection of chemicals for the development and evaluation of in vitro methods for skin sensitisation testing. ATLA 37, 305-312.

Daneshian, M., Akbarsha, M. A., Blaauboer, B. J. et al. (2011). A framework program for the teaching of alternative methods (replacement, reduction, refinement) to animal experimentation. ALTEX 28, 341-352. http://dx.doi.org/10.14573/ altex.2011.4.341

dos Santos, G. G., Reinders, J., Ouwehand, K. et al. (2009). Progress on the development of human in vitro dendritic cell based assays for assessment of the sensitizing potential of a compound. Toxicol Appl Pharmacol 236, 372-382. http:// dx.doi.org/10.1016/j.taap.2009.02.004

ECHA (2014). The Use of Alternatives to Testing on Animals for the REACH Regulation. Second report under Article 117 (3) of the REACH Regulation. ECHA-14-A-07-EN.

EPA (2009). Non-animal testing approach to epa labeling for eye irritation. Office of Pesticide Programs. http://www.epa. gov/pesticides/regulating/eye-policy.pdf

Gerberick, F., Vassallo, J., Foertsch, L. et al. (2007). Quantification of chemical peptide reactivity for screening contact allergens. Toxicol Sci 97, 417-427. http://dx.doi.org/10.1093/ toxsci/kfm064

Gerberick, F., Aleksic, M., Basketter, D. et al. (2008). Chemical reactivity measurement and the predictive identification of skin sensitisers. The report and recommendations of ECVAM workshop 64. ATLA 36, 215-242.

Gibbs, S., Corsini, E., Spiekstra, S. W. et al. (2013). An epidermal equivalent assay for identification and ranking potency of contact sensitizers. Toxicol Appl Pharmacol 272, 529-541. http://dx.doi.org/10.1016/j.taap.2013.07.003

Guzelian, P. S., Victoroff, M. S., Halmes, N. C. et al. (2005). Evidence-based toxicology: a comprehensive framework for causation. Hum Exp Toxicol 24, 161-201. http://dx.doi. org/10.1191/0960327105ht517oa
Hartung, T., Bremer, S., Casati, S. et al. (2004). A modular approach to the ECVAM principles on test validity. ATLA 32, 467-472.

Hartung, T. and Rovida, C. (2009). Chemical regulators have overreached. Nature 460, 1080-1081. http://dx.doi. org/10.1038/4601080a

Hartung, T., Luechtefeld, T., Maertens, A. and Kleensang, A. (2013a). Food for thought ... integrated testing strategies for safety assessments. ALTEX 30, 3-18. http://dx.doi. org/10.14573/altex.2013.1.003

Hartung, T., Stephens, M. and Hoffmann, S. (2013b). Mechanistic validation. ALTEX30,119-130.http://dx.doi.org/10.14573/ altex.2013.2.119

Hoffmann, S. and Hartung, T. (2006). Toward an evidencebased toxicology. Hum Exp Toxicol 25, 497-513. http://dx.doi. org/10.1191/0960327106het648oa

Hooyberghs, J., Schoeters, E., Lambrechts, N. et al. (2008). A cell-based in vitro alternative to identify skin sensitizers by gene expression. Toxicol Appl Pharmacol 231, 103-111. http://dx.doi.org/10.1016/j.taap.2008.03.014

Jacobs, M. N., Laws, S. C., Willett, K. et al. (2013). In vitro metabolism and bioavailability tests for endocrine active substances: what is needed next for regulatory purposes? ALTEX 30, 331-351. http://dx.doi.org/10.14573/altex.2013.3.331

Jaworska, J. and Hoffmann, S. (2010). Integrated Testing Strategy (ITS) - opportunities to better use existing data and guide future testing in toxicology. ALTEX 27, 231-242. http://www. altex.ch/All-issues/Issue.50.html?iid=121\&aid=1

Jaworska, J., Dancik, Y., Kern, P. et al. (2013). Bayesian integrated testing strategy to assess skin sensitisation potency: From theory to practice. J Appl Toxicol 33, 1353-1364.

Johansson, H., Albrekt, A. S., Borrebaeck, C. A. and Lindstedt, M. (2013). The GARD assay for assessment of chemical skin sensitizers. Toxicol. In Vitro 27, 1163-1169. http://dx.doi. org/10.1016/j.tiv.2012.05.019

Judson, R., Kavlock, R., Martin, M. et al. (2013). Perspectives on validation of high-throughput assays supporting $21^{\text {st }}$ century toxicity testing. ALTEX 30, 51-56. http://dx.doi. org/10.14573/altex.2013.1.051

Kavlock, R., Chandler, K., Houck, K. et al. (2012). Update on EPA's ToxCast program: Providing high throughput decision support tools for chemical risk management. Chem Res Tox 25, 1287-1302. http://dx.doi.org/10.1021/tx3000939

Kinsner-Ovaskainen, A., Akkan, Z., Casati, S. et al. (2009). Overcoming barriers to validation of non-animal partial replacement methods/Integrated Testing Strategies: The report of an EPAA-ECVAM workshop. ATLA 37, 437-444.

Kinsner-Ovaskainen, A., Maxwell, G., Kreysa, J. et al. (2012). Report of the EPAA-ECVAM workshop on the validation of Integrated Testing Strategies (ITS). ATLA 40, 175-181.

Kinsner-Ovaskainen, A., Prieto, P., Stanzel, S. and Kopp-Schneider, A. (2013). Selection of test methods to be included in a testing strategy to predict acute oral toxicity: An approach based on statistical analysis of data collected in phase 1 of the ACuteTox project. Toxicol In Vitro 27, 1377-1394. http:// dx.doi.org/10.1016/j.tiv.2012.11.010 
Kleinstreuer, N. C., Judson, R. S., Reif, D. M. et al. (2011). Environmental impact on vascular development predicted by high throughput screening. Environ Health Perspect 119, 1596-1603. http://dx.doi.org/10.1289/ehp.1103412

Knudsen, T. B. (2012). ToxCast and Virtual Embryo: In vitro data and in silico models for predictive toxicology. In T. Seidle and H. Spielmann (ed.), AXLR8-3 Alternative Testing Strategies; Progress Report 2012 (193-200). Springer-Verlag, Berlin, Germany.

Kolle, S. N., Basketter, D. A., Casati, S. et al. (2013). Performance standards and alternative assays: Practical insights from skin sensitisation. Regul Toxicol Pharmacol 65, 278-285. http://dx.doi.org/10.1016/j.yrtph.2012.12.006

Kopp-Schneider, A., Prieto, P., Kinsner-Ovaskainen, A. and Stanzel, S. (2013). Design of a testing strategy using nonanimal based test methods: lessons learnt from the ACuteTox project. Toxicol In Vitro 27, 1395-1401. http://dx.doi. org/10.1016/j.tiv.2012.08.016

Leist, M., Hasiwa, N., Rovida, C. et al. (2014). Consensus report on the future of animal-free systemic toxicity testing. ALTEX 31,341-356. http://dx.doi.org/10.14573/altex.1406091

Martin, M. T., Knudsen, T. B., Reif, D. et al. (2011). Predictive model of rat reproductive toxicity from ToxCast high throughput screening. Biol Reprod 85, 327-339. http://dx.doi. org/10.1095/biolreprod.111.090977

Maxwell, G., Aeby, P., Ashikaga, T. et al. (2011). Skin sensitisation: The Colipa strategy for developing and evaluating nonanimal test methods for risk assessment. ALTEX 28, 50-55. http://dx.doi.org/10.14573/altex.2011.1.050

Maxwell, G., MacKay, C., Cubberley, R. et al. (2014). Applying the skin sensitisation adverse outcome pathway (AOP) to quantitative risk assessment. Toxicol In Vitro 28, 8-12. http:// dx.doi.org/10.1016/j.tiv.2013.10.013

Natsch, A. (2010). The Nrf2-Keap1-ARE toxicity pathway as a cellular sensor for skin sensitizers - functional relevance and a hypothesis on innate reactions to skin sensitizers. Toxicol Sci 113, 284-292. http://dx.doi.org/10.1093/toxsci/kfp228

Natsch, A., Bauch, C., Foertsch, L. et al. (2011). The intra-and inter-laboratory reproducibility and predictivity of the KeratinoSens assay to predict skin sensitisers in vitro: Results of a ring-study in five laboratories. Toxicol In Vitro 25, 733-744. http://dx.doi.org/10.1016/j.tiv.2010.12.014

Natsch, A., Ryan, C. A., Foertsch, L. et al. (2013). A dataset on 145 chemicals tested in alternative assays for skin sensitization undergoing prevalidation. J Appl Toxicol 33, 1337-1352.

Natsch, A. (2014). Integrated approaches to safety testing: General principles and skin sensitization as test case. Issues In Toxicology, 265-288.

Nendza, M., Gabbert, S., Kühne, R. et al. (2013). A comparative survey of chemistry-driven in silico methods to identify hazardous substances under REACH. Regul Toxicol Pharm 66, 301-314. http://dx.doi.org/10.1016/j.yrtph.2013.05.007

NIH (1999). The Murine Local Lymph Node Assay: A Test Method for Assessing the Allergic Contact Dermatitis Potential of Chemicals/Compounds. The Results of an Independent Peer Review Evaluation Coordinated by the Interagency
Coordinating Committee on the Validation of Alternative Methods (ICCVAM)and the National Toxicology Program Center for the Evaluation of Alternative Toxicological Methods (NICEATM). NIH Publication No. 99-4494.

Norlen, H., Worth, A. P. and Gabbert, S. (2014). A tutorial for analysing the cost-effectiveness of alternative methods for assessing chemical toxicity: The case of acute oral toxicity prediction. ATLA 42, 115-127.

NRC - National Research Council, Committee on Toxicity Testing and Assessment of Environmental Agents (2007). Toxicity Testing in the $21^{\text {st }}$ Century: A Vision and a Strategy. Washington, DC, USA: The National Academies Press.

OECD No 168 (2012a). The Adverse Outcome Pathway for Skin Sensitisation Initiated by Covalent Binding to Proteins Part 1: Scientific Evidence. Series on Testing and Assessment No.168. http:/www.oecd.org/officialdocuments/ publicdisplaydocumentpdf/?cote=env/jm/mono(2012)10/ part1\&doclanguage $=$ en

OECD No 168 (2012b). The Adverse Outcome Pathway for Skin Sensitisation Initiated by Covalent Binding to Proteins Part 2: Use of the AOP to Develop Chemical Categories and Integrated Assessment and Testing Approaches. Series on Testing and Assessment No.168. http://www.oecd.org/officialdocuments/publicdisplaydocumentpdf/?cote=env/jm/ mono(2012)10/part2\&doclanguage $=$ en

OECD No 203 (2014). New guidance document on an integrated approach on testing and assessment (iata) for skin corrosion and irritation. Series on Testing and Assessment No. 203. http://www.oecd.org/officialdocuments/publicdisplaydocum entpdf/?cote $=$ env $/ \mathrm{jm} / \mathrm{mono}(2014) 19 \&$ doclanguage=en

OECD TG 428 (2004). Skin Absorption: In Vitro Method. http://dx.doi.org/10.1787/9789264071087-en.

Patlewicz, G., Aptula, A. O., Roberts, D. W. and Uriarte, E. (2008). A minireview of available skin sensitization (Q) SARs/expert systems. QSAR Comb Sci 27, 60-76. http:// dx.doi.org/10.1002/qsar.200710067

Piersma, A. H., Bosgra, S., van Duursen, M. B. et al. (2013). Evaluation of an alternative in vitro test battery for detecting reproductive toxicants. Reprod Toxicol 38, 53-64. http:// dx.doi.org/10.1016/j.reprotox.2013.03.002

Prieto, P., Kinsner-Ovaskainen, A., Stanzel, S. et al. (2013). The value of selected in vitro and in silico methods to predict acute oral toxicityin a regulatory context: Results from the European Project ACuteTox. Toxicol In Vitro 27, 1357-1376. http://dx.doi.org/10.1016/j.tiv.2012.07.013

Python, F., Goebel, C. and Aeby, P. (2007). Assessment of the U937 cell line for the detection of contact allergens. Toxicol Appl Pharmacol 220, 113-124. http://dx.doi.org/10.1016/j. taap.2006.12.026

Reuter, H., Spieker, J., Gerlach, S. et al. (2011). In vitro detection of contact allergens: Development of an optimized protocol using human peripheral blood monocyte-derived dendritic cells. Toxicol In Vitro 25, 315-323. http://dx.doi. org/10.1016/j.tiv.2010.09.016

Richter, A., Schmucker, S. S., Esser, P. R. et al. (2013). Human $\mathrm{T}$ cell priming assay (hTCPA) for the identification of 
contact allergens based on naive T cells and DC-IFN- $\gamma$ and TNF- $\alpha$ readout. Toxicol In Vitro 27, 1180-1185. http://dx.doi. org/10.1016/j.tiv.2012.08.007

Rorije, E., Aldenberg, T., Buist, H. et al. (2013). The OSIRIS Weight of Evidence approach: ITS for skin sensitisation. Regul Toxicol Pharm 67, 146-156. http://dx.doi.org/10.1016/j. yrtph.2013.06.003

Rovida, C. and Hartung, T. (2009). Re-evaluation of animal numbers and costs for in vivo tests to accomplish REACH legislation requirements. ALTEX 26, 187-208. http://www. altex.ch/All-issues/Issue.50.html?iid=107\&aid=4

Rovida, C., Martin, S. F., Vivier, M. et al. (2013). Advanced tests for skin and respiratory sensitization assessment. ALTEX 30, 231-252. http://dx.doi.org/10.14573/altex.2013.2.231

Sakaguchi, H., Ashikaga, T., Miyazawa, M. et al. (2009). The relationship between CD86/CD54 expression and THP-1 cell viability in an in vitro skin sensitization test - human cell line activation test (h-CLAT). Cell Biol Toxicol 25, 109-126. http://dx.doi.org/10.1007/s10565-008-9059-9

Scott, L., Eskes, C., Hoffmann, S. et al. (2010). A proposed eye irritation testing strategy to reduce and replace in vivo studies using bottom-up and top-down approaches. Toxicol In Vitro 24, 1-9. http://dx .doi.org/10.1016/j.tiv.2009.05.019

Smirnova, L., Hogberg, H. T., Leist, M. and Hartung, T. (2014). Developmental neurotoxicity - challenges in the $21^{\text {st }}$ century and in vitro opportunities. ALTEX 31, 129-156. http://dx.doi. org/10.14573/altex.1403271

Sogorb, M. A., Pamies, D., de Lapuente, J. et al. (2014). An integrated approach for detecting embryotoxicity and developmental toxicity of environmental contaminants using in vitro alternative methods. Toxicol Lett 230, 356-367. http://dx.doi. org/10.1016/j.toxlet.2014.01.037
Tluczkiewicz, I., Batke, M., Kroese, D. et al. (2013). The OSIRIS Weight of Evidence approach: ITS for the endpoint repeated-dose toxicity (RepDose ITS). Regul Toxicol Pharm 67, 157-169. http://dx.doi.org/10.1016/j.yrtph.2013.02.004

Van der Veen, J. W., Pronk, T. E., van Loveren, H. and Ezendam, J. (2013). Applicability of a keratinocyte gene signature to predict skin sensitizing potential. Toxicol In Vitro 27, 314 322. http://dx.doi.org/10.1016/j.tiv.2012.08.023

Van der Veen, J. W., Rorije, E., Emter, R. et al.(2014). Evaluating the performance of integrated approaches for hazard identification of skin sensitizing chemicals. Regul Toxicol Pharmacol 69, 371-379. http://dx.doi.org/10.1016/j.yrtph.2014.04.018

Van Loveren, H., Cockshott, A., Gebel, T. et al. (2008). Skin sensitisation in chemical risk assessment: Report of a WHO/ IPCS international workshop focusing on dose-response assessment. Regul Toxicol Pharmacol 50, 155-199. http:// dx.doi.org/10.1016/j.yrtph.2007.11.008

Yoon, M., Campbell, J. L., Andersen, M. E. and Clewell, H. J. (2012). Quantitative in vitro to in vivo extrapolation of cellbased toxicity assay results. Crit Rev Toxicol 42, 633-652. http://dx.doi.org/10.3109/10408444.2012.692115

\section{Correspondence to}

\section{Costanza Rovida}

CAAT Europe

University of Konstanz

Box 600

78457 Konstanz, Germany

Phone: +39 3404008118

e-mail: costanza.rovida@uni-konstanz.de 